\title{
On the Design and Optimization of Micro-Fluidic Dielectrophoretic Devices: A Dynamic Simulation Study
}

\author{
Haibo Li ${ }^{1}$ and Rashid Bashir ${ }^{1,2, *}$ \\ ${ }^{1}$ School of Electrical and Computer Engineering, Purdue University, \\ West Lafayette, IN 47907-1285, USA \\ ${ }^{2}$ Department of Biomedical Engineering, Purdue University, West \\ Lafayette, IN 47907-1285, USA \\ E-mail: bashir@ecn.purdue.edu
}

\begin{abstract}
Microfabricated interdigitated electrode array is a convenient form of electrode geometry for dielectrophoretic trapping of biological particles within micro-fluidic biochips. We have previously reported experimental results and finite element modeling of the holding forces for both positive and negative dielectrophoretic traps on microfabricated interdigitated electrodes within a microfluidic biochip fabricated in silicon with a $12 \mu \mathrm{m}$ deep chamber and anodic-bonded glass cover. Based on these prior studies, we present in this paper a dynamic study to investigate the stopping capability of dielectrophoretic devices with limited electrode teeth. Simulation results on the issues of design and optimization of the dielectrophoretic devices are also presented and discussed in detail. Simulation results show that the maximum particle stopping distance in a specific device is very sensitive to the chamber height due to the near-electrode nature of DEP force. The relationship between maximum stopping distance and the applied voltage is presented, and the electrode spacing is found to be important in designing the electrode geometry. The spacing should be no less than the chamber height in order to efficiently capture the particles in a relatively short range at a given applied voltage and flow rate.
\end{abstract}

Key Words. dielectrophoresis, interdigitated electrode, micro-fluidic, biochip

\section{Introduction}

When biological particles are placed in a non-uniform AC electrical field, they are subjected to dielectrophoretic force resulting from the interaction of the induced electrical polarization charge with the non-uniform electric field. Depending on the particle polarisability compared with the suspending medium, the particle moves either towards the location with the greatest electric field gradient (positive DEP), or location away from the highest electric field gradient (negative DEP) (Pohl, 1978). DEPbased techniques have been successfully used for many biological applications to date, such as separations of viable and nonviable yeast cells (Huang et al., 1992; Markx et al., 1994), separation of live and heat-treated listeria bacteria (Li and Bashir, 2002), isolation and detection of sparse cancer cells, concentration of cells from dilute suspensions, and trapping and positioning of individual cells for characterization (Wang et al., 1997). Among these, the simplest method of practical dielectrophoretic sepa- ration is that of flow separation by using microfabricated interdigitated electrode array at the bottom of the microfluidic devices (Hughes, 2002). So far most studies used a flat, horizontal micro-channel that was formed by a spacer ( $\sim 400 \mu \mathrm{m}$ height is normally used) sandwiched between the bottom electrode plate and a top glass plate. It is known that the magnitude of DEP force reduces quickly with distance above the electrodes, and as a consequence the particles will experience appreciable dielectrophoretic forces only in the area near the electrodes (Markx and Pethig, 1995). Thus the devices with large chamber heights are not suitable for DEP trapping, since not only the particles experiencing negative DEP can't be stopped, some particles experiencing positive DEP have to pass over the electrodes at distance too large for the dielectrophoretic force to be sufficient to trap them.

We have previously reported experimental results and finite element modeling of the holding forces for both positive and negative dielectrophoretic traps on microfabricated interdigitated electrodes within a microfluidic device ( $\mathrm{Li}$ et al., 2005). This device was fabricated by $\mathrm{KOH}$ anisotropically etching a thin $(\sim 12 \mu \mathrm{m})$ micro-channel into single crystalline silicon substrate and the channel was closed with a glass cover using anodic bonding. The schematic plot of the device including the channel and interdigitated microelectrodes are shown in Figure 1. The releasing voltages were measured for different particles (polystyrene beads, yeast cells, spores and bacteria) against destabilizing fluid flows at a given frequency. A simulation environment was developed using Finite Element and numerical methods to obtain near-electrodeplane high-order DEP forces and other forces on particles in the fluidic flow in both horizontal and vertical directions. The experimental results and those from modeling are found to be in close agreement, validating our ability to model the dielectrophoretic filter. However, this information is not sufficient since the releasing voltage is measured in a static case where only force equilibrium is considered. To model real cases where particles enter the DEP-Flow

\footnotetext{
${ }^{*}$ Corresponding author.
} 

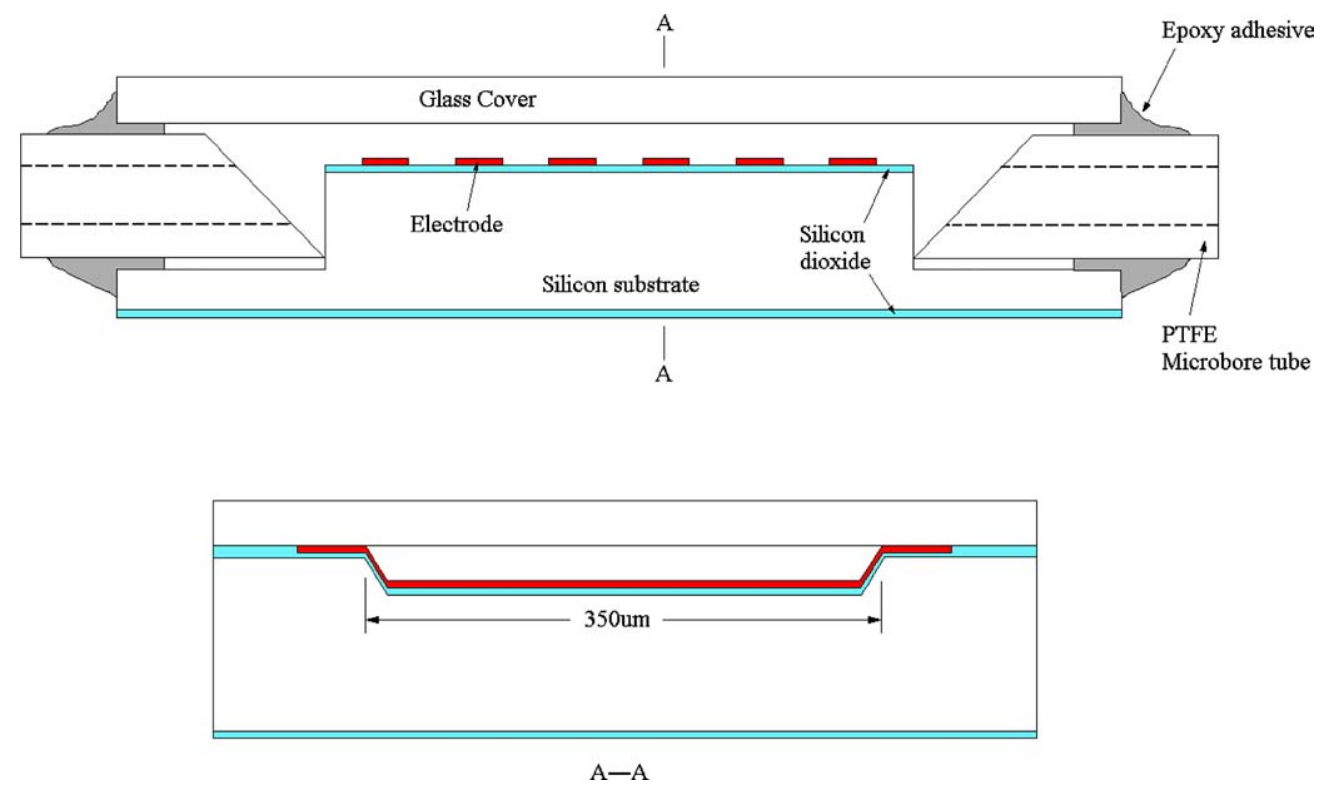

Fig. 1. A schematic plot of the device cross section in (a) and a cross-section along A-A of the chamber in (b).

chamber with varying initial velocities and heights, in this paper we present a dynamic study to evaluate the stopping capability of a dielectrophoretic device with a limited number of electrodes. Along with this, optimization of parameters such as chamber height and electrode array geometry on the design of such dielectrophoretic devices is also discussed.

Particles in the dielectrophoretic filter experience forces from dielectrophoresis, gravity, hydrodynamic drag and lifting effects. The multi-order dielectrophoretic force was given by Washizu and Jones (Jones and Washizu, 1996; Washizu and Jones, 1994) as:

$$
\vec{F}_{\text {total }}=\sum_{0}^{\infty} \vec{F}_{n}=\sum_{0}^{\infty}-\nabla U_{n}
$$

where $n$ refers to the force order, and

$U_{n}=-\frac{2 \pi \varepsilon_{m} K_{n} r^{(2 n+1)}}{(2 n+1) ! !} \sum_{i+j+k=n} \frac{1}{i ! j ! k !}\left[\frac{\partial^{n} \Phi}{\partial x^{i} \partial y^{j} \partial z^{k}}\right]^{2}$

where $\Phi$ is the electrostatic potential of the external electric field, $K_{n}$ is the $n$ th-order Clausius-Mossotti factor

$$
K_{n}=\frac{n(2 n+1)\left(\varepsilon_{p}^{*}-\varepsilon_{m}^{*}\right)}{n \varepsilon_{p}^{*}+(n+1) \varepsilon_{m}^{*}}
$$

where $\varepsilon_{p}^{*}$ and $\varepsilon_{m}^{*}$ are the relative complex permittivities of the particle and the medium respectively and are each given by $\varepsilon^{*}=\varepsilon+\sigma /(j \omega)$, where $\varepsilon$ is the permittivity and $\sigma$ is the conductivity of the particle or medium, and $j$ is $\sqrt{-1}$. Higher-order terms must be taken into account when the small particle is close to the electrode edges or at field nulls for accurate simulations.

The hydrodynamic drag force on a particle of radius $r$ is dependent on the velocity of the particle relative to that of the fluid medium and is given by the modified Stokes equation (Goldman et al., 1967)

$$
\vec{F}_{\text {HD-drag }}=6 \pi k r \eta\left(\vec{v}_{m}-\vec{v}_{p}\right)
$$

where $\eta$ is the dynamic viscosity of the fluid, $\vec{v}_{p}$ and $\vec{v}_{m}$ are the velocity vectors of the particle and the medium fluid at the center of the particle, $k$ is a non-dimensional factor accounting for the wall effects $(k>1$, for particle in contact with the wall $k \approx 1.7$ ). The fluid is usually assumed to follow a parabolic laminar flow profile such that $v$ at a distance $y$ from the bottom of the chamber is:

$$
v=6\langle v\rangle \frac{y}{h}\left(1-\frac{y}{h}\right)
$$

where $\langle v\rangle$ is the mean velocity of the flow and $h$ is the top to bottom spacing of the chamber walls:

$$
\langle v\rangle=\frac{V}{w h}
$$

where $w$ is the width and $w h$ is the cross section area of the chamber, $V$ is the nominal flow rate in $\mu 1 / \mathrm{min}$. The sedimentation force is given by:

$$
F_{\text {sedi }}=\frac{4}{3} \pi r^{3}\left(\rho_{p}-\rho_{m}\right) g
$$

where $r$ is the radius of the particle, $\rho_{p}$ and $\rho_{m}$ refer to the densities of the particle and medium respectively, and $g$ is the gravitational acceleration constant. The Hydrodynamic lifting force experienced by a non-deformable 
particle located at a small distance $y-r$ above the chamber wall is given by:

$$
\left.F_{\text {lift }} \approx 0.153 r^{3} \eta \frac{1}{(y-r)} \cdot \frac{d v_{m}}{d y}\right|_{y=0}
$$

This formula was empirically derived by (Williams et al., 1992) but the nature of the lift force still remains in question. Calculation shows that this force is much smaller than the dielectrophoretic force and previous study showed it plays little or no role in typical DEP-Gravitational FieldFlow process (Wang et al., 1998), it is still incorporated into our modeling in order to get a complete view of the force field.

The modeling Matlab (R12, The Mathworks, Natick, MA) program used electric-field data from commercially available Finite Element program Ansys (version 5.7, ANSYS Inc. Canonsburg, PA) with grid spacing as small as $0.2 \mu \mathrm{m}$. Since the electrodes are long compared to their width, the problem can be considered to be twodimensional. For a given particle and flow rate, we calculated the DEP forces in both horizontal and vertical directions to an arbitrary order until sufficient accuracy was obtained (the result converges with relative error less than $3 \%$ ) in the sedimentation force, and the hydrodynamic drag force everywhere in space. The moving path of the particle in the chamber was obtained by solving the following ordinary differential equations for particle position $(x(t), y(t))$ with initial conditions of the particle position and velocity when entering the chamber:

$$
\left\{\begin{array}{l}
F_{x}=m v_{x}^{\prime}=\operatorname{DEP}_{x}(x, y)+6 \pi k r \eta\left[v_{m}(y)-v_{x}\right] \\
F_{y}=m v_{y}^{\prime}=\operatorname{DEP}_{y}(x, y)-6 \pi k r \eta v_{y} \\
\quad-\frac{4}{3} \pi r^{3}\left(\rho_{p}-\rho_{m}\right) g+\left.0.153 r^{3} \eta \frac{1}{(y-r)} \cdot \frac{d v_{m}}{d y}\right|_{y=0}
\end{array}\right.
$$

where subscript $x$ and $y$ indicate the directions. Both dielectrophoretic forces $\mathrm{DEP}_{x}$ and $\mathrm{DEP}_{y}$ are functions of position $(x, y)$ and were obtained by linear interpolation from the calculated data at the grid points. The boundary condition would be:

$$
v_{y}=\left.0\right|_{y=0, y=h}
$$

Here the particle velocity in $y$ direction is assumed to be zero after particle collides with the chamber bottom or top. Friction between particle and chamber wall is neglected.

\section{Results and Discussion}

First, we consider that spherical particles with radius $1 \mu \mathrm{m}$ are injected into a $12 \mu \mathrm{m}$-high DEP chamber with isosce- les trapezoidal cross-section (top width $350 \mu \mathrm{m}$ ) and bottom electrode array with $20 \mu \mathrm{m}$ electrode width and 20 $\mu \mathrm{m}$ spacing at flow rate $0.1 \mu \mathrm{l} / \mathrm{min}$ (corresponding to a velocity of $\sim 186.4 \mu \mathrm{m} / \mathrm{s}$ at the center of the particle in contact with the top or bottom of the chamber, or a mean velocity of the flow $\langle v\rangle=406.7 \mu \mathrm{m} / \mathrm{s}$ ). The suspending medium is DI water with conductivity $1 \times 10^{-4} \mathrm{~S} / \mathrm{m}$ and viscosity $1 \times 10^{-3} \mathrm{~N} \cdot \mathrm{s} / \mathrm{m}^{2}$, and the real part of the polarization factor of the particles is assumed to be $\sim 0.43$ (positive DEP, for example, for bacteria) at $1 \mathrm{MHz}$. Figure 2 shows the moving paths of particles at different initial heights in the chamber with voltage $5 \mathrm{~V}_{\mathrm{pp}}$ applied to the electrodes (release voltage is $3.24 \mathrm{~V}_{\mathrm{pp}}$ ). The bold lines at the horizontal axis represent the electrodes. The particles exhibit horizontal projectiles but with downward acceleration greater than $g$, especially near the electrode surface. The particle tracks have kinks when passing the electrode edges and stop at the electrode edges eventually. This is expected from the fact that the maximum DEP forces exist at the electrode edges.

Figure 3 shows the corresponding horizontal stopping distance for particles entering the chamber in Figure 2 with different initial heights. It is seen that a particle with the maximum initial height $(11 \mu \mathrm{m})$ will travel horizontally about $230 \mu \mathrm{m}$ before it stops at one of the electrode edges at $5 \mathrm{~V}_{\mathrm{pp}}$. This is to say, in order to capture all the particles injected into the chamber, the electrode array length must be at least $\sim 230 \mu \mathrm{m}$, which corresponds to 6 electrode teeth, if the applied voltage is $5 \mathrm{~V}_{\mathrm{pp}}$.

Next, we consider the effect of the applied voltage on the maximum stopping distance of the particles. As shown in Figure 4, as the applied voltage approaches the release voltage $\left(3.24 \mathrm{~V}_{\mathrm{pp}}\right)$, the maximum stopping distance of particles (corresponding to that of particle with maximum initial height) increases rapidly, and the particle can be stopped at the first electrode when the voltage is increased to $\sim 13 \mathrm{~V}_{\mathrm{pp}}$. Careful considerations must be made when designing the DEP electrode array length and maximum applied voltage at a desired flow rate, since a very high voltage, and hence high electric field, can be detrimental to the particles (bacteria and cells), and the induced electrohydrodynamic effect can be significant, especially at low frequency (Green and Morgan, 1998).

Chamber height is another important parameter to be designed. Since it is well-known that the DEP force decreases exponentially with height, we wouldn't be surprised to see that with a small increase in chamber height, the maximum particle stopping distance will increase dramatically. For comparison, we consider the DEP chamber as discussed above with only the change in chamber height. We change the flow rate according to the changed chamber cross-section-area so as to keep the same mean velocity of the flow $\langle v\rangle=406.7 \mu \mathrm{m} / \mathrm{s}$, applied voltage is still $5 \mathrm{~V}_{\mathrm{pp}}$ (release voltage ranges from 3.52 to $2.56 \mathrm{~V}_{\mathrm{pp}}$ 


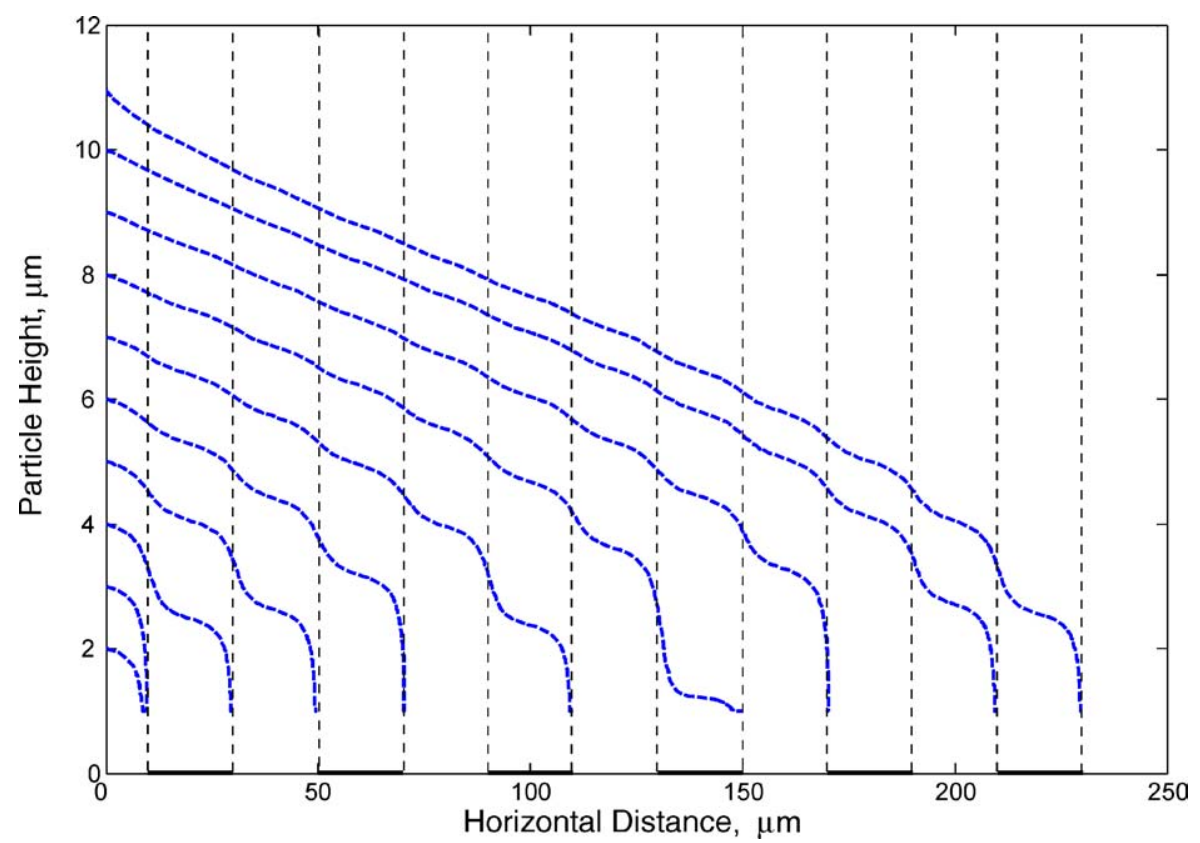

Fig. 2. Moving path of particle at different initial heights in $12 \mu \mathrm{m}$ chamber with $20 \mu \mathrm{m} / 20 \mu \mathrm{m}$ electrode with/spacing, flow rate $0.1 \mu \mathrm{l} / \mathrm{min}$, applied voltage $5 V_{p p}$ (release voltage is $3.24 V_{p p}$ ).

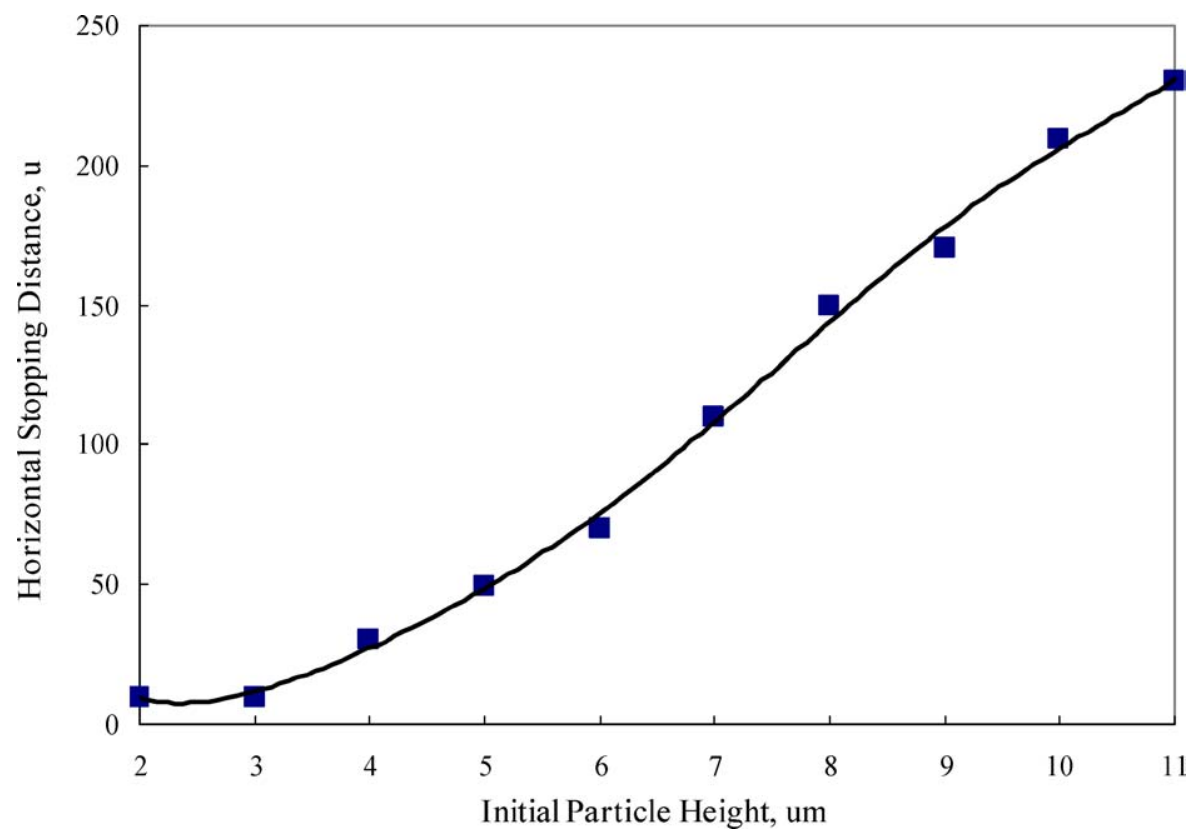

Fig. 3. Trendline of the horizontal stopping distance for particles at different initial heights in $12 \mu \mathrm{m}$ chamber with $20 \mu \mathrm{m} / 20 \mu \mathrm{m}$ electrodes, flow rate $0.1 \mu \mathrm{l} / \mathrm{min}$, applied voltage $5 V_{p p}$ (release voltage is $3.24 V_{p p}$ ).

for chamber height from 10 to $20 \mu \mathrm{m}$, due to different fluidic velocity profiles). Figure 5 confirms our prediction. Moreover, under the above conditions, the maximum particle stopping distance increases from $\sim 150$ to $\sim 870 \mu \mathrm{m}$ as the chamber height changes from 10 to $20 \mu \mathrm{m}$.
The final device parameter investigated in this paper is the electrode array geometry, including electrode width and spacing. In general, as the electrode spacing decreases, the DEP force increases at the same applied voltage, so that the capture capability of electrode array with smaller spacing will be higher. However, this is an "unfair" 


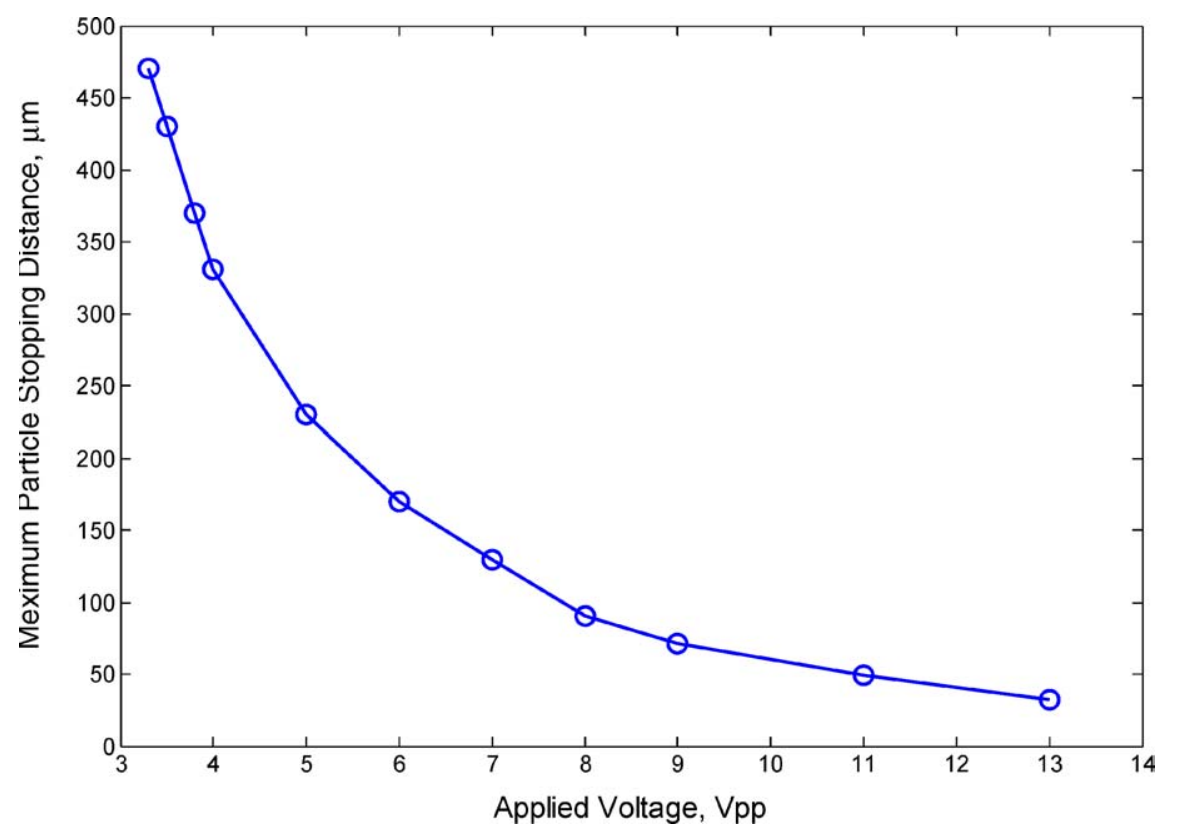

Fig. 4. Maximum particle stopping distances at different applied voltage on the $20 \mu \mathrm{m} / 20 \mu \mathrm{m}$ electrodes in $12 \mu \mathrm{m}$ chamber, particle initial height is $11 \mu \mathrm{m}$, flow rate $0.1 \mu \mathrm{l} / \mathrm{min}$ (release voltage is $3.24 \mathrm{~V}_{p p}$ ).

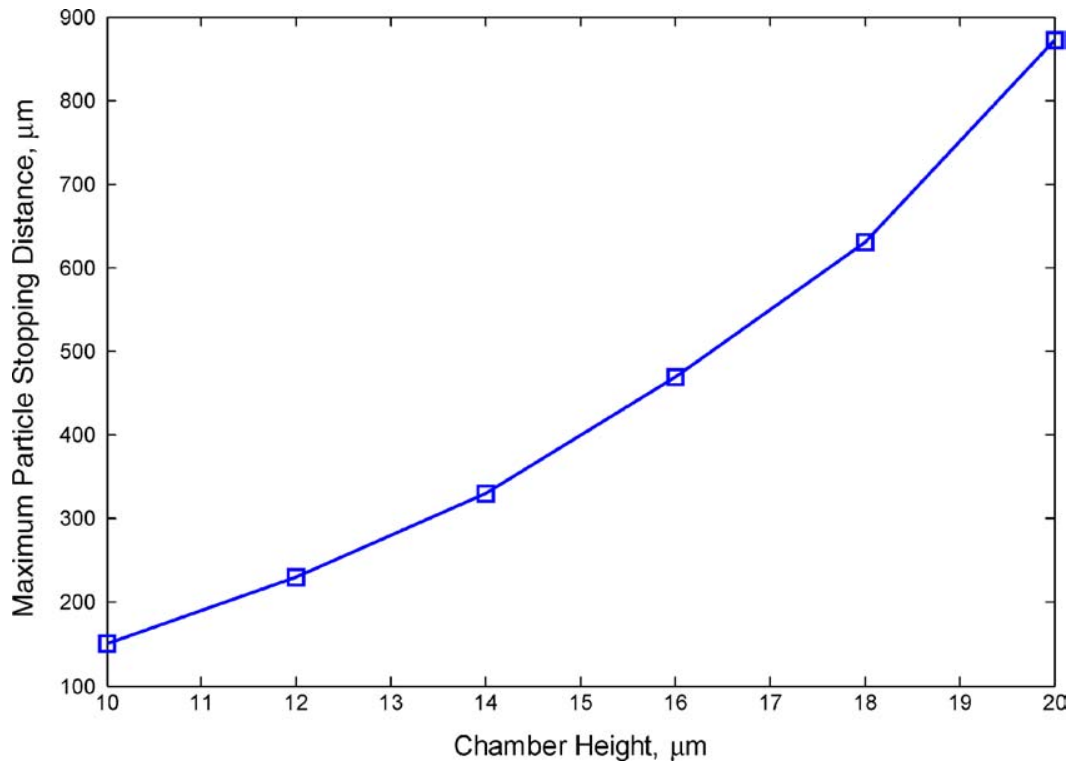

Fig. 5. Maximum particle stopping distances in chambers with different heights. The electrodes are $20 \mu \mathrm{m}$ wide with $20 \mu \mathrm{m}$ spacing, under the same mean velocity of the flow $\langle v\rangle=406.7 \mu \mathrm{m} / \mathrm{s}$, applied voltage $5 V_{p p}$ (release voltage ranges from $3.52 V_{p p}$ to $2.56 V_{p p}$ due to different fluidic velocity profiles).

criterion in comparing the capture capabilities of electrode arrays with different spacing, since higher voltage can always be used in an electrode array with larger spacing to obtain the same DEP force. For comparability, we should apply different voltages to electrode arrays with different spacing but keep the nominal electric field the same to all electrode arrays. The nominal electric field is defined as the applied voltage over the electrode spacing. Figure 6 shows the release voltages (left $y$ axis) and corresponding nominal electric field strengths (right $y$ axis) for the same particle in $12 \mu \mathrm{m}$ chambers with electrode arrays having different spacing but total width + spacing $=40 \mu \mathrm{m}$ at flow rate of $0.1 \mu \mathrm{l} / \mathrm{min}$, from a viewpoint of force equilibrium. It can be seen that the release voltage increases slowly as the spacing increases and then decreases, with a maximum located at spacing $\sim 28 \mu \mathrm{m}$, while the 


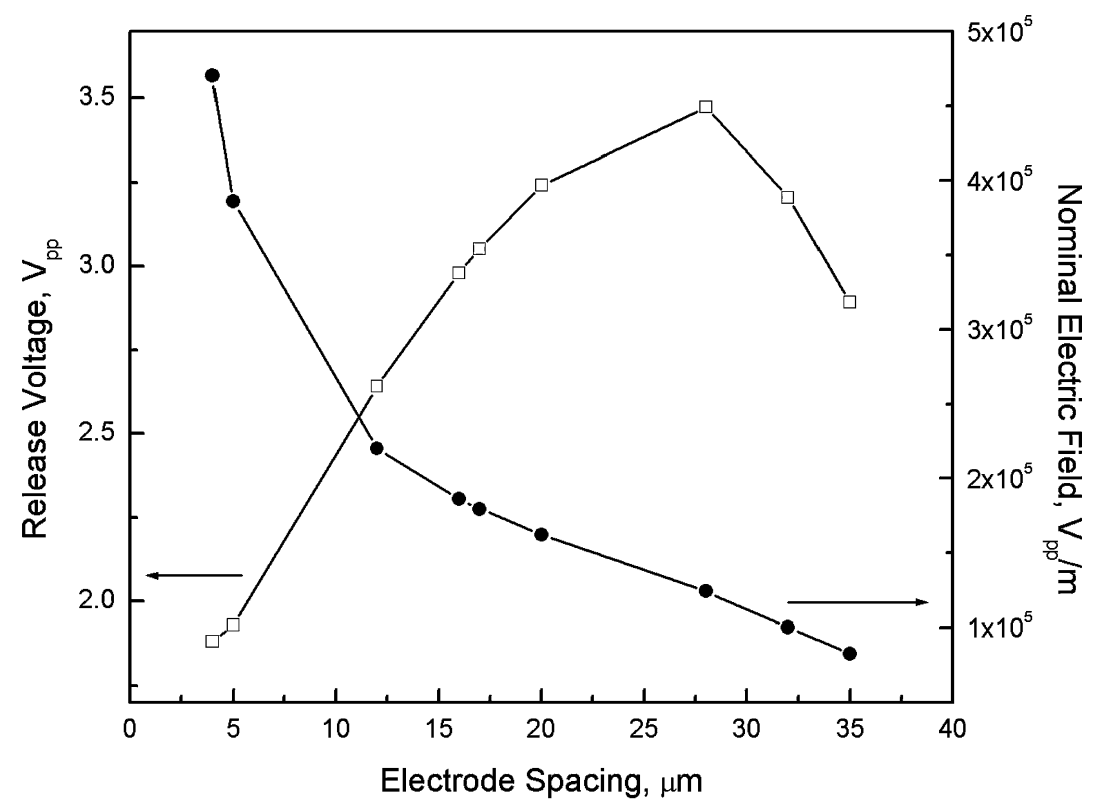

Fig. 6. The release voltages (left y axis) and corresponding nominal electric field strengths (right y axis) for the particles injected into $12 \mu \mathrm{m}$ chamber with electrode arrays having different spacing but total width + spacing $=40 \mu \mathrm{m}$, at flow rate of $0.1 \mu \mathrm{l} / \mathrm{min}$.

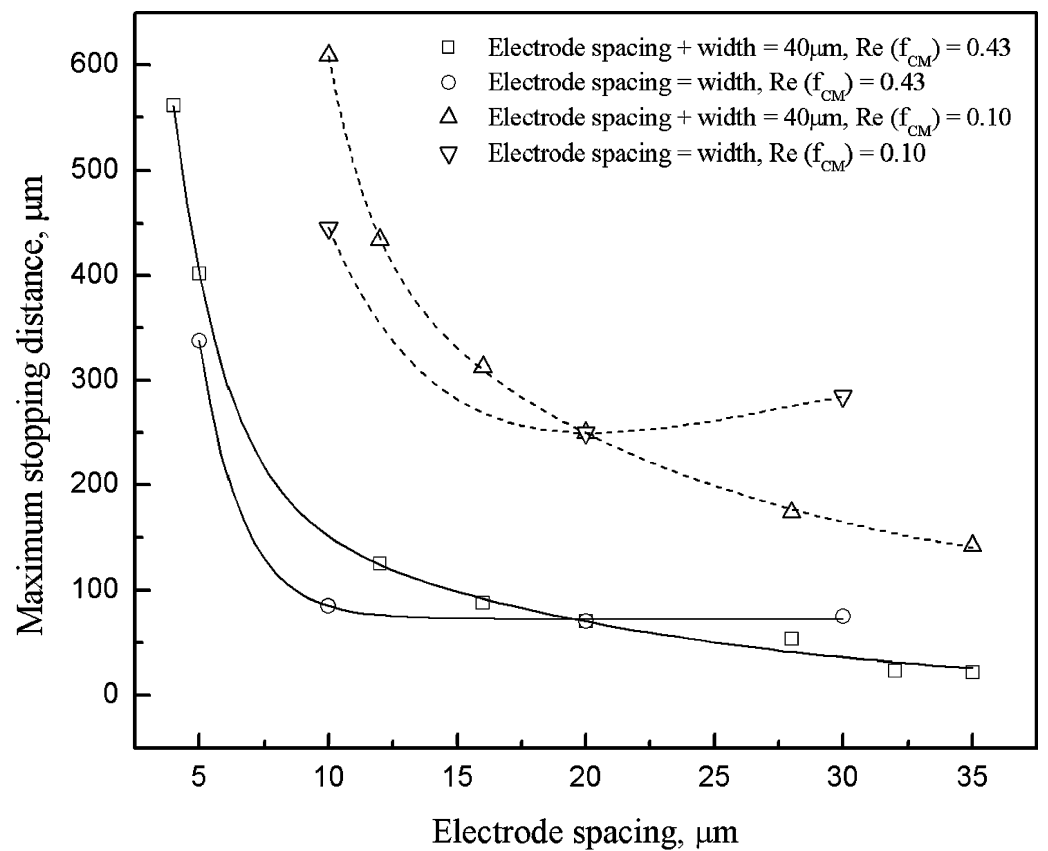

Fig. 7. Maximum particle stopping distances in $12 \mu \mathrm{m}$ chambers with different electrode widths and spacing but same nominal electric field $5 \times 10^{5}$ $V_{p p} / \mathrm{m}$ at flow rate of $0.1 \mu \mathrm{l} / \mathrm{min}$ for particles with real part of the polarization factor of 0.43 and 0.10 .

corresponding nominal electric field strength keeps decreasing. The decrease of the release voltage is due to the change of the electric field profile as the electrodes become narrower, which results in higher electric field gradient and DEP force. It turns out that the electrode array with larger spacing would have higher capture capability if voltage were applied to keep the same nomi- nal electric field strength; yet very narrow electrode width is limited by the photolithographic process at the bottom of the chamber.

Consistent with the above conclusion, a dynamic simulation shows in Figure 7 the maximum particle stopping distances versus electrode spacing for different electrode arrays with total width + spacing $=40 \mu \mathrm{m}$ in these 
$12 \mu \mathrm{m}$ chambers at the same nominal electric field $5 \times 10^{5}$ $\mathrm{V}_{\mathrm{pp}} / \mathrm{m}$ and flow rate of $0.1 \mu \mathrm{l} / \mathrm{min}$. It can be seen that the maximum stopping distance decreases rapidly with the increased spacing for spacing smaller than the chamber height. Similar trend is also found for electrode array with equal width and spacing as shown in Figure 7. This reveals that the spacing between electrodes is the most important design consideration when seeking a desired chamber length and operational voltage and the general rule is that the spacing should be no less than that of chamber height.

It is also of great interest to look at the effect of particle polarization factor on the stopping distance since it is directly related to the DEP force. The relationship between the maximum stopping distance and electrode spacing is also included in Figure 7 for particles with real part of the polarization factor 0.1 and under the same other conditions. Because of the reduced DEP force, the release voltage has to increase for the same flow rate, and the particles can't be stopped at the same nominal electric field for small electrode spacing (for example, release voltage changes from 1.93 to $3.93 \mathrm{~V}_{\mathrm{pp}}$ for particles on electrode array with $35 \mu \mathrm{m}$ width and $5 \mu \mathrm{m}$ spacing, and from 1.49 to $3.05 \mathrm{~V}_{\mathrm{pp}}$ for particles on electrode array with $5 \mu \mathrm{m}$ width and $5 \mu \mathrm{m}$ spacing, particles can't be stopped at $2.5 \mathrm{~V}_{\mathrm{pp}}$, which corresponds to the nominal electric field of $5 \times 10^{5} \mathrm{~V}_{\mathrm{pp}} / \mathrm{m}$ ). The maximum stopping distance approaches infinite quickly when the electrode spacing is smaller than $10 \mu \mathrm{m}$. It can be seen that the polarization condition of the particle is also important in designing the device and electrode geometries for particle trapping in positive DEP. Adjusting the medium conductivity and operating frequency can be used to maximize the particle polarization factor. It should be noted that, though the results in this papers are from simulations and haven't been confirmed by rigorous experiments, they are based on the modeling environment confirmed by our experiments of dielectrophoretic trapping described in (Li et al., 2005) and should be capable of being a general guide for the micro-fluidic dielectrophoretic device design.

\section{Conclusion}

In this paper we present a dynamic study to investigate the capture capability of dielectrophoretic devices with different geometries based on our established simulation environment. Simulation results show that the maximum particle stopping distance in a specific device is very sensitive to the chamber height due to the near-electrode nature of DEP force. The relationship between maximum stopping distance and the applied voltage is presented, and the electrode spacing is found to be important in designing the electrode geometry. The spacing should be no less than the chamber height in order to efficiently capture the particles in a relatively short range at a given applied voltage and flow rate.

\section{Acknowledgment}

This research was supported through a cooperative agreement with the Agricultural Research Service of the United States Department of Agriculture, project number 193542000-035 through the Center for Food Safety Engineering at Purdue University.

\section{References}

A.J. Goldman, R.G. Cox, and H. Brenner, Chemical Engeering Science 22, 653-660 (1967).

N.G. Green and H. Morgan, Journal of Physics D: Applied Physics 31, L25-L30 (1998).

Y. Huang, R. Holzel, R. Pethig, and X.-B. Wang, Phys. Med. Biol. 37, 1499-1517 (1992).

M.P. Hughes, Electrophoresis 23, 2569-2582 (2002).

T.B. Jones and M. Washizu, Journal of Electrostatics 37, 121-134 (1996).

H. Li and R. Bashir, Sensors and Actuators B: Chemical 86, 215-221 (2002).

H. Li, Y. Zheng, D. Akin, and R. Bashir, IEEE Journal of Microelectromechanical Systems, to appear (2005).

G.H. Markx and R. Pethig, Biotechnology and Bioengineering 45, 337343 (1995).

G.H. Markx, M.S. Talary, and R. Pethig, Journal of Biotechnology 32, 29-37 (1994).

H.A. Pohl, Dielectrophoresis (Cambridge University Press, Cambridge, 1978).

X.-B. Wang, Y. Huang, P.R.C. Gascoyne, and F.F. Becker, IEEE Transactions on Industry Applications 33, 660-669 (1997).

X.-B. Wang, J. Vykoukal, F.F. Becker, and P.R.C. Gascoyne, Biophysical Journal 74, 2689-2701 (1998).

M. Washizu and T.B. Jones, Journal of Electrostatics 33, 187-198 (1994).

P.S. Williams, T. Koch, and J.C. Giddings, Chemical Engineering Communications 111, 121-147 (1992). 